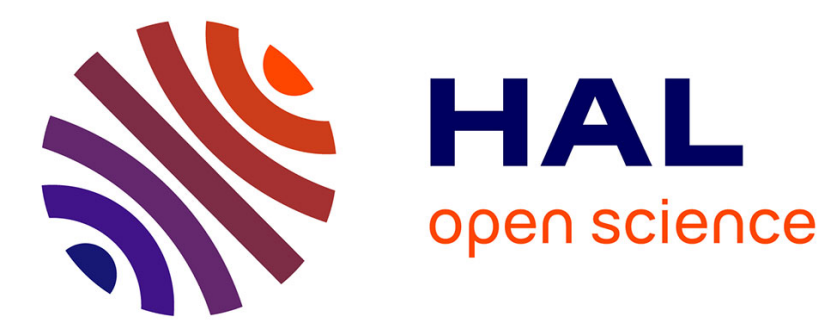

\title{
Gouvernance régionale, innovation et mouvements sociaux dans le Québec contemporain : état de la situation
}

Denyse Côté, Étienne Simard

\section{- To cite this version:}

Denyse Côté, Étienne Simard. Gouvernance régionale, innovation et mouvements sociaux dans le Québec contemporain: état de la situation . Quebec Studies, 2012, 54, pp.111-125. hal-01528862

\section{HAL Id: hal-01528862 \\ https://hal.science/hal-01528862}

Submitted on 11 Jul 2017

HAL is a multi-disciplinary open access archive for the deposit and dissemination of scientific research documents, whether they are published or not. The documents may come from teaching and research institutions in France or abroad, or from public or private research centers.
L'archive ouverte pluridisciplinaire HAL, est destinée au dépôt et à la diffusion de documents scientifiques de niveau recherche, publiés ou non, émanant des établissements d'enseignement et de recherche français ou étrangers, des laboratoires publics ou privés. 
Gouvernance régionale, innovation, et mouvements sociaux dans le Québec contemporain: état de la situation

\author{
Denyse Côté \\ Université du Québec en Outaouais \\ Étienne Simard \\ Université du Québec en Outaouais
}

\title{
Introduction
}

L'arrivée en scène de luttes sociales articulées par des mouvements combatifs et diversifiés dans la deuxième moitié du vingtième siècle est à l'origine de nombreuses réformes et innovations sociales ${ }^{1}$ au Québec. En effet, le Québec connut une Révolution tranquille dès 1960 qui modernisa l'appareil gouvernemental ainsi que l'économie québécoise et enclencha une série de réformes sociales permettant de créer un véritable État-providence. Basé sur un nationalisme renouvelé, inspiré de la vague d'affirmations nationales des pays du Sud, cette dixième province canadienne, berceau de la survivance francophone en Amérique du Nord, commença alors à se considérer comme un État à part entière, bien qu'aucune réforme constitutionnelle n'ait été opérée. Suite à ces transformations menées par les élites et permettant l'émergence d'une bourgeoisie québécoise francophone et d'une population mieux scolarisée et plus articulée, un mouvement populaire, étudiant, syndical et féministe émergea, inspiré du "printemps" étudiant de 1968. Réprimé en 1970 lors de la "Crise d'octobre," il en resta néanmoins un "mouvement populaire autonome" qui constitue à ce jour une réelle force politique avec lesquels ont dû conjuguer les gouvernements successifs.

Ces mouvements ont laissé derrière eux des structures organisationnelles qui ont contribué à un meilleur partage des richesses ainsi qu'à l'élargissement de la démocratie à des zones auparavant laissées dans l'ombre (Fraser 2005). S'appuyant sur la reconnaissance et le financement de l'État qu'elles ont réussi à obtenir, les organisations issues de ces mouvements se sont consolidées, institutionnalisées, et regroupées en fédérations. Elles quadrillent maintenant le Québec, intervenant sur une base territoriale aussi bien que sur des problèmes spécifiques (santé, logement, endettement, violences sexuelles, etc.) liés à toutes les sphères de la vie sociale (Côté et Simard 2011).

Dans les régions québécoises, ${ }^{2}$ les mécanismes de gouvernance propres à ce que certains ont nommé le "modèle québécois" (Côté, Lévesque, et Morneau 2007) ont permis un arrimage de ces organisations communautaires aux instances de planification du développement économique et social apparues subséquemment en région. La reconnaissance de l'expertise des groupes communautaires par le gouvernement du Québec a assuré leur permanence dans l'espace public mais a eu par la même occasion un impact sur leurs dynamiques internes et externes, sur leurs rôles et missions. Dans ce contexte, les mouvements sociaux ont-ils toujours le même 
potentiel d'innovation? Les mécanismes de concertation ${ }^{3}$ avec l'appareil gouvernemental en milieu régional pourraient-ils freiner l'émergence d'innovations issues de mouvements sociaux? Pour répondre à ces questions, nous examinerons l'arrimage des organisations communautaires aux nouveaux mécanismes de concertation régionale à l'aide de données de recherches menées auprès d'organisations communautaires dans sept régions québécoises ainsi que d'observations recueillies au cours d'expériences professionnelles et participatives réparties sur une trentaine d'années. L'objectif étant ici d'appréhender cette situation complexe à partir de données recueillies de première main et de la littérature québécoise en particulier, d'en faire sens et d'analyser sa portée sans pour cela procéder, faute d'espace, à un exposé empirique.

\section{Mouvements sociaux et structures issus de la Révolution tranquille}

L'État québécois des années 1960 s'est construit par des réformes sociales successives dans un élan de "modernisation accélérée sur le modèle de l'État-providence" (Ferretti 1999) que l'on a nommé "Révolution tranquille": nationalisation de l'électricité, réforme de l'éducation et de la santé, création du Conseil d'orientation économique du Québec et de la Caisse de dépôt et de placements, etc. À cette époque le nationalisme politique florissant sert de véhicule aux aspirations de la population et des élites politiques pour un changement social d'envergure. Cet élan s'essoufflera à l'aube des années 1980 non sans avoir apporté d'importants changements au paysage sociopolitique, aux structures et aux orientations du Gouvernement $\mathrm{du}$ Québec et qui persistent à ce jour. Il aura par la même occasion donné naissance à de nouveaux mouvements sociaux (Vaillancourt 1992) inspirés au départ du mouvement des droits civils aux États-Unis, des mouvements étudiants en Europe et en Amérique du Nord et de libération nationale africains et latino-américains.

Ces nouveaux mouvements sociaux, communautaire, féministe, et étudiant québécois se sont consolidés et ont exercé des pressions continues pour améliorer les conditions de vie des secteurs défavorisés. En réponse, le gouvernement a progressivement mis en place des pratiques et structures de reconnaissance des groupes issus de ces mouvements ainsi que de leurs innovations sociales. Un mode de financement a été créé dès la fin des années 1970 et une reconnaissance de leur expertise d'intervention auprès des groupes exclus a été obtenue par la suite (Gouvernement du Québec 1988). Le processus d'institutionnalisation des groupes communautaires et de leurs pratiques innovatrices ( $D^{\prime}$ amours 2000; Lévesque et Vaillancourt 1998; Bourque 1997) sont uniques au monde et méritent qu'on s'y arrête.

En effet, cette institutionnalisation prend différentes formes et comprend différentes phases:

[les] règles de compromis [émergent] entre les acteurs [et portent sur] le financement, les conditions de développement, les [modalités] de participation, les principes de démocratisation, les formes d'ententes partenariales, les politiques sociales, etc. (D'amours 2000, 2) 
Le premier type d'institutionnalisation de groupes communautaires est celui de la création d'institutions publiques inspirées d'innovations communautaires. Des groupes innovent en réponse aux besoins d'une collectivité locale. Ces innovations sont par la suite transformées en services sociaux publics et reproduits sur l'ensemble du territoire par l'appareil gouvernemental. Il s'agit habituellement de services de proximité: ce fut le cas des Centres locaux de services communautaires (CLSC), des garderies, des services d'aide juridique ainsi que des services d'aide aux entrepreneurs maintenant offerts par les Centres locaux de développement (CLD). Comme le soulignent Lévesque et Vaillancourt (1998), l'ensemble des règles à l'intérieur des structures nouvellement créées échappent aux acteurs et groupes qui auront été à l'origine du compromis leur ayant donné naissance.

Dans d'autres cas, la reconnaissance gouvernementale permet de maintenir l'autonomie structurelle des organismes communautaires. La Politique de reconnaissance des organismes communautaires autonomes (PRSAC) du gouvernement du Québec y réfère, lorsqu'elle reconnaît la

spécificité des organismes communautaires autonomes par rapport à l'économie sociale et au mouvement coopératif; [le respect de] l'autonomie des organismes communautaires en s'éloignant d'une complémentarité et d'un partenariat obligés avec l'État; [la centralité du] soutien à la mission comme mode de financement le plus susceptible de favoriser l'émergence d'une participation citoyenne innovatrice émanant d'un milieu où les problèmes sont directement vécus. (Guay et White 2009, 20)

L'institutionnalisation se traduit alors plutôt à travers un financement public récurrent donnant lieu progressivement à des mandats précis. Des programmes (Renaud et al. 1997) ou ententes de services (Tardif 2007) deviennent alors avec le temps partie intégrante des organisations communautaires, "de [leur] mission, de [leur] organigramme [...] et des budgets [annuels]" (Renaud et al. 1997, 109). Ces groupes communautaires normalisent en quelque sorte leur dépendance financière à l'État qui, à son tour, exerce une influence grandissante sur leurs pratiques et leur culture organisationnelles. L'institutionnalisation des groupes communautaires se traduit aussi sur le plan de la légitimité que lui confèrent les pouvoirs publics: le gouvernement du Québec reconnaît que certains organismes représentent la population qu'ils desservent et les invite à siéger aux côtés du patronat et des syndicats au sein d'instances de concertation (Lévesque 2004; Observatoire du Québec 2002; Bourque 2000; Dupuis 1998). Ces mécanismes de concertation constituent une des assises du "modèle québécois," ${ }^{\prime 4}$ qui s'est graduellement arrimé au néolibéralisme ambiant (Bouchard, Lévesque, et St-Pierre 2005).

Issu de la Révolution tranquille, ce modèle de concertation entre les pouvoirs publics québécois et certaines organisations issues de la société civile renvoie aussi au corporatisme social québécois des années 1930, 
lorsque l'Église exerçait sa gouverne sur les composantes organisationnelles de la société civile. ${ }^{5}$ L'État y jouait alors un rôle de médiateur permettant, dans le cas des relations de travail par exemple, "[d'harmoniser les] intérêts entre le patronat et les travailleurs à l'intérieur des corps intermédiaires" (Côté, Lévesque, et Morneau 2007, 11). La laïcisation subséquente des institutions québécoises a suscité, selon certains auteurs, l'émergence d'un nouveau type de corporatisme ${ }^{6}$ en matière de relations de travail (Observatoire du Québec 2002, 18; Archibald 1983), transformant les syndicats et le patronat en "partenaires conflictuels" au sein de l'État, destinés à s'organiser sur leurs propres bases, à travailler ensemble à la coconstruction d'une économie nationale, mais à faire appel à l'État "pour définir un cadre institutionnel qui permet la négociation des demandes sociales et la régulation des conflits, à l'exemple des pays scandinaves" (Côté, Lévesque, et Morneau 2007, 11). Selon certains auteurs (Bacqué, Rey, et Sintoner 2005; D’Amours 2000; Hamel 1989; Bélanger 1988), il s'agit d'un modèle de néo-corporatiste. Au tournant du vingt-et-unième siècle, les pressions des mouvements sociaux québécois ont permis d'intégrer de plus en plus d'acteurs collectifs (associations, organismes communautaires, etc.) à ces processus de concertation dessinés à l'origine pour les syndicats (Lévesque, Bourque, et Vaillancourt 1999, 6). Plusieurs groupes communautaires ont donc été invités à siéger au sein d'instances permanentes de consultation régionale ou provinciale ou de forums sociaux et économiques dits de "concertation," dans le but d'assurer la représentation de leur secteur d'intervention.

La participation à de tels mécanismes de concertation a légitimé plusieurs organisations communautaires et leur a permis d'occuper l'espace public sur une base plus permanente. Cette légitimité octroyée par les pouvoirs publics a graduellement remplacé la légitimité issue directement de leurs bases respectives, les organismes communautaires centrant de plus en plus leurs activités sur les besoins de l'appareil, qu'il soit gouvernemental ou communautaire, plutôt que sur la mobilisation des populations marginalisées, laissant alors peu de place à leur propre renouvellement ou au renouvellement des demandes issues des groupes exclus (Shragge 2003).

\section{Régionalisation, gouvernance, et groupes communautaires en région}

Parallèlement, rappelons que le processus étapiste de décentralisation amorcé il y a trente ans par le gouvernement québécois a été désigné sous le vocable de "régionalisation." Au gré de l'adoption de politiques en matière de développement économique, social, et culturel, des responsabilités et pouvoirs ont été graduellement dévolus aux dix-sept régions administratives québécoises, dans l'optique de la transformation du rôle de l'État "providence" et "planificateur" en État "accompagnateur" (Bourque 2000; Klein 1995). Ils ont été confiés à des organismes intermédiaires créés par le gouvernement: les Conférences régionales des élus (CRÉ). Celles-ci sont structurées sur le mode d'un organisme non gouvernemental. Contrairement aux villes et aux municipalités, elles ne disposent d'aucun pouvoir de taxation ou mécanisme électif; elles sont uniquement imputables au gouvernement qui les a créées. Elles sont toutefois responsables de la gestion du 
développement socio-sanitaire et économique de chacune des régions québécoises. Le gouvernement central leur reconnaît une représentativité et une expertise et leur délègue le mandat de concerter les acteurs régionaux et de financer les projets de développement à envergure régionale. Se mettent alors en place progressivement dans chaque région du Québec une toile complexe d'instances décisionnelles et de concertation. Y participent à la fois des élus et des représentants d'organismes pourvoyeurs de services ou experts dans des secteurs jugés prioritaires. Ces organismes entretiennent des liens entre eux, au sein de ces instances de gouvernances régionales.

Cette régionalisation constituait à l'origine la réponse gouvernementale au mouvement régionaliste des années soixante et soixante-dix opposé à la centralisation de l'État-providence québécois alors en émergence. Ce régionalisme était arrimé à la fois à des mouvements sociaux régionaux et aux intérêts et discours identitaires et économiques des élites régionales. En réponse à ces demandes, de même qu'à la nécessité d'assurer le développement économique de l'ensemble de son territoire, le gouvernement du Québec a entamé ce processus de régionalisation par la création de Conseils régionaux de la Santé et des services sociaux, de Conférences administratives régionales, et de Conseils régionaux de la Culture. Par la suite, différentes politiques de développement économique régional se sont succédées: le "Choix des régions" en 1983, “Le Québec à l'heure de l'entreprise régionale" en 1988, etc. Ces politiques ont souvent eu recours à la concertation avec la société civile, sous le règne du Parti Québécois en particulier. La réforme Picotte de 1993 (Gouvernement du Québec 1992) implante des Conseils régionaux du développement $(\mathrm{CRD})^{7}$ dans chacune des régions administratives du Québec; leurs pouvoirs sont confirmés et élargis par la réforme Michaud de 1997. À partir de ce moment, le concept de régionalisation au Québec renverra toujours à des mécanismes de gouvernance ${ }^{8}$ faisant appel à la société civile (Lévesque 2004, 10).

En 2003, le gouvernement libéral implante un modèle qu'il prétend en rupture avec la Révolution tranquille et qui répondrait aux impératifs du néolibéralisme (Lévesque 2004, 11). La loi $34^{9}$ promulguée en 2004 crée des Conférences régionales des élus (CRÉ) qui remplaceront les CRD. Il s'agit $\mathrm{d}^{\prime}$ organismes intermédiaires ${ }^{10}$ à but non lucratif et incorporés dont l'existence, la composition, et le mandat sont déterminés par une loi provinciale, mais dont la gestion est autonome et qui sont imputables à leur conseil d'administration qui prend acte des directives décentralisées par Québec. Contrairement aux municipalités, ils n'ont pas de pouvoir de taxation ou de légitimité représentative, les membres de leur conseil d'administration étant désignés par la loi ou cooptés. Les CRÉ sont les interlocuteurs privilégiés du gouvernement du Québec pour leur région respective avec mandat de planifier son développement et de gérer les budgets accordés au développement de leur région ${ }^{11}$ par celui-ci.

Contrairement aux CRD dont le mandat se limitait au développement économique, les CRÉ se sont fait confier par Québec un mandat élargi en matière de développement social. Parallèlement, la place de la société civile dans les processus décisionnels s'est rétrécie en termes de nombre de voix au conseil d'administration et de disparition des mécan- 
ismes électifs. En effet, la loi 34 accorde une place centrale (au moins deux tiers des sièges) dans les conseils d'administration des CRÉ aux préfets des Municipalités régionales de comté (MRC) qui ne sont pas élus, ou encore à des conseillers municipaux élus sur un mandat local plutôt que régional par une fraction seulement de la population régionale. ${ }^{12}$ Désormais, plutôt que de participer à des tables de concertation inter-organisationnelles et inter-sectorielles, les organisations se voient intégrées à des structures contrôlées par des élus qui ne sont redevables à aucun électorat régional.

Les pouvoirs conférés à une majorité d'élus locaux d'une région leur donne la possibilité de se contenter "d'enregistrer la présence des forces organisées" et éventuellement de sélectionner les plus pertinentes par rapport à l'espace public concerné et d'écarter celles qui s'élèveraient contre l'ordre constitutionnel (Baqué, Rey, et Sintoner 2005, 19). Ils auraient ainsi le pouvoir de balayer toute organisation qui voudrait remettre en question le fonctionnement qu'ils proposent. Ceci est d'autant plus vrai que le gouvernement du Québec a octroyé aux CRÉ le pouvoir d'évaluer les organismes méritant un financement public.

Le processus de planification et d'application des plans quinquennaux régionaux mobilise des ressources importantes au sein des groupes communautaires de chaque région et exerce ainsi une influence importante sur leurs orientations. Dans plusieurs cas, les ressources, les orientations et le développement de groupes communautaires ont été soumis aux décisions et projets des CRÉ. Contrairement aux CRD qui privilégiaient plutôt des structures de concertation inter-organisationnelles, les CRÉ semblent ainsi plutôt intégrer les organisations à des structures contrôlées par des élus municipaux et des gestionnaires du développement.

\section{Gouvernance et émergence d'innovations sociales en région}

L'espace public régional québécois devient alors progressivement occupé par le discours émanant des mécanismes de concertation régionale (Côté et Simard 2010). Les agents gouvernementaux s'en font les porte-parole tant au nom des dirigeants que des exclus, restreignant dans les faits cet espace public délibératif aux seules tables de concertation. Ceci constitue un frein à l'émergence d'innovations sociales en région qui ne sont pas conformes aux thèmes, intérêts, ou priorités de ces tables de concertation ou des dirigeants régionaux. Il est en effet connu que les innovations sociales émergent dans bien des cas de mouvements ou de mouvances sociales distinctes et souvent même réactives aux idées, discours, et règles de l'heure.

Or les mécanismes de concertation régionale privilégient ouvertement le modèle de l'entrepreneur créatif (Schumpeter 1976), car leur mode de fonctionnement enchâsse des procédures précises: les discours sur les thèmes de l'exclusion et des droits de la personne (égalité hommes-femmes, itinérance, sécurité alimentaire, etc.) doivent être portés par les organisations communautaires œuvrant dans ces champs et au sein des tables de concertation désignées, ou encore par les porte-parole des instances régionales. Les changements sociaux se restreignent à des projets portés par des promoteurs collectifs dûment identifiés. Ce qui provoque l'exclusion 
de groupes ou d'idées qui n'adopteraient pas ces nouveaux modes de fonctionnement. Or le débat public entre citoyens ou groupes de citoyens concernés, et avec les autorités politiques, se doit dans une société démocratique d'inclure le discours de contre-publics subalternes (Fraser 2005), riche en nouveaux débats sociaux. Ceux-ci ne sont pas nécessairement conformes aux discours des élites locales et régionales siégeant aux CRÉ, et, partant, conformes aux priorités des instances régionales.

L'exclusion se construit au quotidien (McAll 1995). Pour lutter contre celle-ci, en particulier contre les nouvelles formes d'exclusion ainsi que les nouveaux problèmes sociaux, encore faut-il accepter que l'émergence de nouveaux acteurs issus des "contre-publics subalternes" se produise aussi par le truchement de rapports conflictuels avec les élites et les autorités politiques. La voix de ces contre-publics subalternes est portée par des mouvements sociaux émergents en lutte pour prise en compte dans l'espace public. Ainsi, la reconnaissance des droits des démunis, des locataires, des femmes, accidentés du travail, des sans-emploi ne peut se faire concrètement dans la sphère publique sans ces associations dont ils contrôlent la mission et les actions. Celles-ci peuvent alors entrer en relation avec les autorités politiques et économiques afin de faire connaître leurs besoins et intérêts. Il s'agit donc d'une "conduite collective organisée $\mathrm{d}^{\prime}$ un acteur de classe luttant contre son adversaire de classe pour la direction sociale de l'historicité dans une collectivité concrète" (Touraine 1978, 104). En effet, les mouvements sociaux prennent forme lorsque se combinent l'expression concrète de trois éléments (Touraine 1973, 411). En premier lieu, l'identité ou l'autodéfinition de l'acteur collectif, sa conscience qu'il est spécifique (Touraine 1973, 411; Boucher 1992, 9; Lamoureux 1981, 6). Cette identité d'un mouvement social ne peut se définir indépendamment du deuxième élément, celui d'un conflit réel avec un autre acteur identifié (Touraine 1973, 322), qui "fait surgir l'adversaire [et] forme la conscience des acteurs en présence" (Touraine 1973, 362). Enfin, le troisième élément nécessaire à l'émergence d'un mouvement social est celui de la totalité définie comme "champ d'existence commun aux deux adversaires en conflit" (Lamoureux 1981, 7), comme l'enjeu central sur lequel se centralise cette opposition (Boucher 1992, 9), ou encore comme un "système d'action historique dont les adversaires, situés dans la double dialectique des classes sociales, se disputent la domination" (Touraine 1973, 363).

Chaque acteur connaît "une solidarité spécifique et s'oppose à l'autre [autour de] l'appropriation et [de] la destination de valeurs et ressources sociales" (Melucci 1978, 37-38). Le conflit se situe ainsi au centre de la formation d'un mouvement social et s'exprime dans un espace qui déborde les structures et normes politiques ou institutionnelles existantes. Qui plus est, un mouvement social "comprend [...] toutes les conduites qui cassent les normes institutionnalisées dans les rôles sociaux, qui débordent les règles du système politique et/ou qui attaquent la structure des rapports de classes d'une sociétés (Melucci 1978, 38). Un mouvement social

lutte contre un pouvoir qui gère un système de normes et de rôles. L'action vise une répartition différente des ressources, une 
adaptation fonctionnelle de l'organisation, une (nouvelle) distribution des rôles [...]. Elle tend en même temps à casser les limites institutionnelles de l'organisation et à dépasser son cadre normatif. Le conflit dépasse le cadre de l'organisation et se déplace vers le système politique. (Melucci 1978, 38)

Enfin, un mouvement social n'agit pas "strictement à l'intérieur du système politique existant; [il cherche] à le dépasser, en ouvrant des canaux nouveaux pour l'expression de la demande politique, en poussant la participation au-delà des limites [...] prévues" (Melucci 1978, 38-39). Le dépassement du cadre institutionnel et politique de l'heure, sa "non-focalisation sur le système politique" institutionnel serait même une condition inhérente à ce qu'une conduite collective soit considérée comme un mouvement social (Melucci 1978, 49). De cette façon, un mouvement social est en mesure de donner un sens à ses activités et de produire à partir de celleci des orientations sociales et culturelles (Touraine 1974).

Ainsi, l'expression d'un conflit est une condition centrale à l'émergence d'un mouvement social et à la création de propositions nouvelles; il ne peut se constituer que dans un espace extérieur aux structures politiques ou administratives. Or, dans l'espace régional, rien n'est moins sûr car celui-ci est occupé par des structures de concertation régionale au sein desquelles sont intégrées les organisations issues des mouvements sociaux antérieurs: ce cadre décourage en fait la construction d'un espace propre.

La nécessité de contrôler le conflit oblige le système de domination à intervenir constamment sur les différents niveaux de la structure [afin de] les maintenir dans les limites de la compatibilité qui correspondent aux rapports de classe fondamentaux. (Melucci 1978, 45)

Les nouvelles structures régionales ne sont pas des institutions au sens traditionnel du terme, mais plutôt des instances "moitié institutions, moitié appareils" (Laurin-Frenette 1999, 9) dont la régulation est de nature technojuridique (Bourque, Duchastel, et Canet 1999). À l'intérieur de ce cadre, par définition, aucun conflit ouvert n'est possible, "le code génétique des procédures de concertation [...] visant à renouer le lien entre la société civile et le politique empêche toute expression du conflit" (Jouve 2005, 326). En effet, les acteurs au sein des instances de gouvernance régionale agissent dans un espace où le conflit ne peut s'exprimer. De plus, la "reconnaissance institutionnelle par l'État de certains groupes contribue à figer les frontières de ceux-ci, à essentialiser leur identité et à y enfermer les individus qui sont poussés à participer en tant que femme, indigène [sic], etc., pour avoir plus de chance de faire entendre leur voix" (Bacqué, Rey, et Sintomer 2005, 19). Or "si le conflit ne dépasse pas les limites du système politique ou du système organisationnel [en question], on aura plutôt affaire à une compétition politique ou à une opposition d'intérêt à l'intérieur d'un cadre normatif donné" (Melucci 1978, 38) plutôt qu'à un mouvement social. 
La professionnalisation des acteurs de la société civile participant à ces mécanismes de concertation (Côté et Simard 2011; Jouve 2005; Lamoureux 1999) ainsi que la tendance actuelle à l'incorporation de la citoyenneté (Bourque, Duchastel, et Pineault 1999) freinent aussi l'émergence de nouveaux mouvements sociaux en région. Or une société ne peut qu'être produite par elle-même: "les luttes doivent être menées par les acteurs concernés" (Lamoureux 1981, 7). Une société ne peut être produite uniquement par des professionnels. Conçue au départ comme une forme d'intégration des populations défavorisées au développement régional, $\mathrm{I}^{\prime \prime \prime}$ institutionnalisation" des organismes communautaires et leur intégration aux structures de gouvernance régionale peut les éloigner encore plus de leur base: effet pervers, certes, lié au fait que dans la plupart des cas, ce sont des professionnels communautaires plutôt que les citoyens désavantagés eux-mêmes qui mènent la lutte.

Cette tendance au transfert du pouvoir des membres de la base vers les travailleurs avait déjà été soulevée par Godbout (1991). C'est comme si l'idéal de la gestion par la base s'était transformé en idéal d'autogestion (c'est-à-dire de gestion par les producteurs de services) puis en idéal de gestion technocratique (c'est-à-dire de gestion centrée sur les besoins de l'appareil). (Côté et Simard 2011)

Le potentiel d'émergence de nouveaux mouvements sociaux en région peut s'avérer ainsi obstrué par la très grande place qu'occupent ces instances dans l'espace public régional, ainsi que par la négation des mécanismes de construction d'acteurs issus des contre-publics subalternes (Fraser 2005). Ceci est particulièrement vrai en ce qui a trait à la prise de conscience de l'acteur, des enjeux qui se fait souvent dans des espaces extérieurs aux structures existantes, à travers la construction de leviers organisationnels autonomes articulés autour de nouveaux enjeux sociaux et souvent à travers le conflit avec les discours et structures existantes.

\section{Quelle place en région pour les nouveaux mouvements sociaux?}

À travers les canaux des médias nationaux, les organisations communautaires contribuent encore aujourd'hui à vitaliser le débat public et l'exercice d'une démocratie délibérative et ouverte. Les quelques 4000 organisations communautaires québécoises regroupées au sein d'environ 250 fédérations font toujours figure de rempart contre le néolibéralisme ambiant, et font toujours entendre une voix discordante au discours des élites. Cependant, ils exercent aussi, paradoxalement, une influence régulatrice sur les nouvelles mouvances sociales et ce, en douce rupture avec leurs pratiques originelles. Car la reconnaissance et le financement que les groupes communautaires ont gagnés de haute lutte font partie $d^{\prime} u n$ processus $d$ 'institutionnalisation, seul capable d'assurer une durabilité des innovations mises de l'avant par les mouvements sociaux. Et ces organismes communautaires doivent rendre des comptes autant, et même parfois davantage, 
au gouvernement qu'à leur base citoyenne. Dans les faits, ils se structurent désormais très souvent autour de problématiques privilégiées par les politiques et orientations gouvernementales.

En région québécoise, l'espace nécessaire à l'émergence de mouvements sociaux contemporains et à la formulation de nouvelles problématiques sociales se trouve ainsi restreinte par cette omniprésence de groupes communautaires semi-institutionnalisés. Leur intégration aux structures régionales de concertation et de consultation oriente leurs activités et ressources vers un développement économique et social planifié par les CRÉ, plutôt que vers la mobilisation des populations vulnérables qu'ils représentent. L'ensemble organisationnel qui en résulte est complexe, certes plus stable financièrement, professionnalisé, et même partiellement bureaucratisé.

On observe encore parfois l'émergence de groupes, souvent plus radicaux, qui opèrent en retrait des structures de gouvernance, et survivent mieux dans les grands centres urbains. Les effets du nombre et du pouvoir attractif des grandes villes permet toujours le renouvellement et la multiplication d'espaces d'expression diversifiés. En région, les conditions sont souvent moins propices. Les organisations intégrées aux réseaux officiels occupent l'ensemble des espaces délibératifs à l'intérieur des structures de gouvernance. Ils sont également regroupés en fédérations nationales qui voient à la formation, à la coordination, à la recherche, et la représentation politique liés à leur secteur précis d'activité. Au niveau régional, les groupes communautaires qui ont passé le cap des deux ou trois décennies d'existence sont donc à la fois rattachés à ces fédérations et regroupés régionalement. Ils absorbent ainsi la quasi totalité des ressources militantes potentiellement disponibles. L'espace permettant l'identification $\mathrm{d}^{\prime}$ une problématique nouvelle, la définition d'une lutte et l'expression de la critique s'en trouve réduits. Tout nouveau groupe est habituellement référé par les instances régionales ou par les organismes communautaires au groupe déjà financé et cuvrant dans ce champ.

On assiste ainsi à la formalisation d'expertises au sein des groupes communautaires qui se transforment progressivement en seuls représentants reconnus d'un secteur d'activité sociale au sein du système de concertation technocratique. Ceci contribue à empêcher l'émergence ou à marginaliser tout nouveau regroupement formé en dehors de cet espace. Un espace délibératif indépendant des structures de gouvernance, pourtant essentiel à l'expression de conflits entre différents projets de société, est à peu près inexistant. Les nouveaux enjeux sont ainsi soit évacués ou récupérés sans suite réelle par des organismes reconnus.

Dans les régions québécoises, les nouvelles structures mises en place à travers le processus de décentralisation n'auront donc pas permis la création d'un réel espace public délibératif entre gouvernants et gouvernés, ou la production de la distance nécessaire à la discussion des intérêts de tous ses citoyens (Thériault 1996; Habermas 1993). Ceci en particulier pour les contre-publics subalternes (Fraser 2005) qui font face à une régulation accrue. La "compétition" directe avec les réseaux officiels de groupes communautaires qui possèdent plus de ressources et un accès plus direct 
aux tribunes régionales représente un défi de taille pour tout nouveau groupe qui voudrait se faire connaître.

En matière d'innovation, le modèle privilégié par les dispositifs de concertation régionale et mieux adaptés à leur culture n'est pas celui des nouveaux mouvements sociaux, mais plutôt celui proposé par l'économiste Schumpeter (1976). Selon lui, la démocratie serait une compétition entre leaders plutôt qu'un espace de délibération autour du bien commun tel que décrit par Habermas (1993). L'innovation serait la clef du changement économique ou politique et aurait pour force motrice les entrepreneurs (pour l'économie) ou les leaders (pour le politique). Appliqué au développement social en région, cette thèse soutiendrait que ce sont les groupes promoteurs de projets "durables," "finançables," avec des résultats tangibles (mesurables) qui assureraient la vitalité démocratique de la région plutôt que les citoyens eux-mêmes ou les mouvements sociaux qu'ils construisent.

Selon cette conception, l'innovation et le changement social proviendraient de spécialistes plutôt que des citoyens eux-mêmes. Le potentiel d'intervention des citoyens sur leurs propres conditions de vie semble ici exclu. Il est à prévoir que de nouveaux types de réseaux parallèles émergent, permettant l'expression de conduites collectives ignorées par les dispositifs formels de concertation. Car le potentiel créatif des mouvements citoyens reste encore à être pleinement pris en compte par les autorités. Par nature, l'action militante se renouvelle au sein des réseaux parallèles; elle saura sans doute s'adapter à ce nouveau contexte et se construire de nouveaux espaces. Par ailleurs les nouvelles structures régionales de concertation avec les groupes communautaires régionaux permettent $\mathrm{d}^{\prime}$ assurer une meilleure régulation des problématiques sociales sur un territoire. Elles intègrent les demandes d'une écoute portées par les groupes communautaires régionaux depuis plus de quarante ans. Mais elles permettent par la même occasion d'orienter la mission et les actions des groupes qui y participent, et de garder dans l'ombre, et même de limiter le développement de mouvements d'opposition; enfin, elles ne règlent en rien la question de la redistribution du pouvoir.

Si le Québec demeure une référence historique majeure en matière de mouvements sociaux et de gouvernance "partagée," il fait face aujourd'hui à un défi politique et social majeur: celui de l'institutionnalisation de ses mouvements sociaux de première génération. Le message envoyé aux nouveaux militants en région est le suivant: si tu veux participer à un mouvement social de défense des droits, il vaudrait mieux que tu te professionnalises (en cadre de groupe communautaire), que tu te taises, ou que tu te joignes à des mouvements plus radicaux qui ont pignon sur rue, non pas dans ta région d'origine, mais dans la métropole. Car l'espace public régional reste sourd aux voix des nouveaux "contre-publics subalternes." Or la fracture entre riches et pauvres s'élargit au Québec suite à deux décennies de politiques néolibérales et il est impératif que la voix des moins nantis puisse émerger avec force sur la scène politique régionale. En effet, les leaders politiques régionaux, ceux qui, selon Schumpeter, sont 
la source de l'innovation sociale, bien qu'en rapport constant avec les groupes communautaires à travers les structures de concertation de leur région, n'ont pas pour autant adopté les intérêts des démunis comme priorité d'action et d'intervention.

\section{Notes}

1 Selon Harrisson et Vézina $(2006,130)$, "les innovations sociales concernent la coordination des relations entre les acteurs sociaux dévolus à la résolution de problèmes socio-économiques, en vue d'une amélioration des performances et du bien-être des collectivités."

2 Le Québec est divisé en dix-sept régions administratives dont la taille varie entre celle d'une ville (Montréal, Québec) ou d'un territoire plus vaste que celui de la France (Nord-du-Québec). Nous appréhendons la région québécoise "dans sa double dimension d'espace physique et politique et comme "nouveau territoire de référence du politique" (Jouve 2005, 323), comme nouveau lieu de convergence des discours et des pratiques où institutions, secteur privé et groupes communautaires (se trouvent en) proximité" (Côté 1995, 112).

3 La concertation serait "un processus collectif de coordination basé sur une mise en relation structurée et durable entre des acteurs sociaux autonomes qui acceptent de partager de l'information, de discuter de problèmes et d'enjeux spécifiques [...] afin de convenir d'objectifs communs et d'actions" (Bourque 2008, 5). La concertation a été introduite au Québec par le Parti Québécois et utilisée "[...] en vue d'obtenir l'adhésion des acteurs sociaux à des objectifs économiques et sociaux. La participation aux premières rencontres de concertation était tripartite (syndicale, patronale et gouvernementale) mais, sous la pression surtout des groupes de femmes, elle a [...] été étendue à divers groupes communautaires. La concertation repose sur une volonté explicite de s'entendre sur le diagnostic et les solutions d'un problème: les conflits d'intérêts entre les parties y sont donc placés en retrait. Les décisions ne sont pas prises à la suite d'un vote majoritaire: elles doivent émerger d'un consensus." (Piotte 1998, 198). "Si la concertation peut camoufler l'opposition des intérêts entre les parties, elle ne peut la supprimer, pas plus qu'elle ne peut abolir le rapport de forces qui unit les parties" (Piotte 1998, 199).

${ }^{4}$ Un "mode particulier de gestion économique et sociale qui s'est développé au Québec depuis les années 1960 " caractérisé par "le rôle actif de l'État dans la vie économique et le mode particulier de concertation entre les instances gouvernementales et les agents économiques dans l'élaboration des politiques sociales et économiques ainsi que dans la mis en oeuvre de certains programmes de développement" (Observatoire du Québec 2002, 14-15).

${ }^{5}$ Notamment par le biais de la Confédération des travailleurs catholiques du Canada (CTCC), qui avait comme principes qu' "entre ouvriers et patrons peuvent exister des intérêts divergeants mais non fondamentalement opposés" et que "l'État n'est pas un instrument de domination d'une classe sur une autre, il est au service du bien commun" (Favreau et L'Heureux 1984, 20).

${ }^{6}$ Le corporatisme est un "un système de représentation des intérêts dans lequel les unités constituantes sont organisées en un nombre limité de catégories singulières, obligatoires, non-compétitives, reconnues ou agréées - sinon créées - par l'État et auxquelles on a garanti un monopole délibéré de représentation au sein de leurs catégories respectives, en échange de l'observation de certains contrôles sur la sélection des leaders et l'articulation des demandes et des intérêts" (Bourque 1995, 14).

7 Une dizaine de régions avaient déjà leur CRD depuis le début des années 1970.

8 "En règle générale, dans les travaux scientifiques, la gouvernance désigne l'émer- 
gence ou la mise en oeuvre par les pouvoirs publics de nouvelles mesures de concertation ou de partenariat afin de mobiliser les ressources provenant d'acteurs économiques ou d'acteurs sociaux rattachées à la société civile dans le but d'atteindre les finalités que les États modernes ne parviennent plus à réaliser d'une manière isolée" (Hamel 2006, 6).

${ }^{9}$ Loi sur le ministère du Développement économique et régional et de la Recherche, présentée le 12 novembre 2003 et adoptée le 17 décembre de la même année.

${ }^{10}$ Le phénomène n'est pas propre au Québec. D'ailleurs, un nouvel acronyme, "GONGO," a été créé pour désigner ces "GOvernmental Non-Governmental Organizations.

" Par développement régional, on entend aussi bien le développement social que le développement économique.

12 Les Conseils Régionaux de Développement (CRD) qui ont précédé les CRÉ ne devait accorder qu'un tiers de ces sièges aux élus municipaux.

\section{Ouvrages Cités}

Archibald, C. Un Québec corporatiste? Hull: Asticou, 1983.

Bacqué, M.-H., H. Rey, et Y. Sintomer. Gestion de proximité et démocratie participative. Une perspective comparative. Paris: La Découverte, 2005.

Bélanger, P. R. "Les nouveaux mouvements sociaux à l'aube des années 90." Nouvelles pratiques sociales 1.1 (1998): 101-14.

Bouchard, M. J. De l'expérimentation à l'institutionnalisation positive. L'innovation sociale dans le logement communautaire au Québec. Montréal: Cahiers du CRISES, 2005.

Bouchard, M. J., B. Lévesque, et J. St-Pierre. Modèle québécois de développement et gouvernance. Entre le partenariat et le néolibéralisme? Montréal: Cahiers du CRISES. 2005.

Boucher, J. Les mowvements sociaux. Réflexion à partir des théories de l'action collective et de la régulation. Montréal: Cahiers du CRISES, 1992.

Bourque, D. Concertation et partenariat. Québec: PU Québec, 2008.

—. "Trajectoire de l'organisation communautaire professionnelle." Nouvelles pratiques sociales 10.1 (1997): 59-70.

Bourque, G. L. Le modèle québécois de développement. De l'émergence au renouvellement. Québec: PU Québec, 2000.

—. Le néo-corporatisme comme angle d'analyse de la nouvelle politique industrielle au Québec. Montréal: Cahiers du CRISES, 1995.

Bourque, G. L., J. Duchastel, et E. Pineault. "L'incorporation de la citoyenneté." Sociologie et sociétés. 31.2 (1999): 41-64.

Bourque, G. L. et B. Lévesque. Le modèle québécois en question. Montréal: Cahiers du CRISES, 1999.

Coté, D. "Développement communautaire et rapports sociaux de sexe: Essai d'analyse sur toile de fond régionale." Du local au planétaire. Réflexions et pratiques de femmes en développement regional. Éds. D. Côté et al. Montréal: Remueménage, 1995. 109-133.

Côté, D. et Ê. Simard. "De l'utopie radicale à la bonne gouvernance: Le cas du Québec." AmeriQuests 7.1 (2010): 42-53. 
-. "Local Governance and Social Movements in Québec: The Perverse Effects of Corporate Culture." Human Dignity and Managerial Responsibility. Eds. D. Crowther and A.-M. Davila-Gomez. London: Gower, 2011. 75-95.

Côté, L., B. Lévesque, et G. Morneau. "L'évolution du modèle québécois de gouvernance: Le point de vue des acteurs." Politique et sociétés 26.1 (2007): 3-26.

D'Amours, M. Procès d'institutionnalisation de l'économie sociale au Québec. Montréal: Cahiers du CRISES, 1999.

Dupuis, J.-P. "Les rôles des acteurs locaux et régionaux dans la construction du modèle québécois de développement économique." Territoires et développement économique. Éd. M-U. Proulx. Paris: L'Harmattan, 1998.

Favreau, L. et P. L'Heureux. Le projet de société de la CSN de 1966 à aujourd'hui. Montréal: Centre de formation populaire, 1984.

Ferretti, L. "La Révolution tranquille," L'Action nationale 89.10 (1999): 59-91.

Fraser, N. Qu'est-ce que la justice sociale? Reconnaissance et distribution. Paris: La Découverte, 2005.

Gouvernement du Québec. Rapport de la Commission d'enquête sur les services de santé et les services sociaux. Québec: Les Publications du Québec, 1988.

-. Développer les régions du Québec. Québec: OPDQ, 1992.

Guay, L. et D. White. "Une politique novatrice sous observation." Revue Relations 731 (mars): 19-21.

Habermas, J. L'espace public. Archéologie de la publicité comme dimension constitutive de la société bourgeoise. Paris: Payot, 1993.

Hamel, P. "Développement local et action communautaire à l'heure du néo-corporatisme." L'autre économie. Une économie alternative. Éds. B. Lévesque, A. Joyal, et O. Chouinard. Québec: PU Québec, 1989. 261-76.

Hamel, P. et B. Jouve. Un modèle québécois? Gouvernance et participation dans la gestion publique. Montréal: PU Montréal, 2006.

Harrisson, D. et M. Vézina. "L'innovation sociale: Une introduction." Annals of Public and Cooperative Economics 77.2 (2006): 129-38.

Jouve, B. "La démocratie en métropoles: Gouvernance, participation et citoyenneté." Revue française de science politique 55.2 (2005): 317-37.

Lamoureux, D. "Mouvement social et lutte des femmes." Sociologie et sociétés 13.2 (1981): 131-38.

Lamoureux, J. La concertation. Perspectives théoriques sous l'angle du néo-corporatisme. Montréal: Cahiers du CRISES, 1996.

Laurin-Frenette, N. "Le démantèlement des institutions intermédiaires de la régulation sociale: Vers une nouvelle forme de domination." Sociologie et societies 31.2 (1999): 65-72.

Lemieux, V. "La révolution tranquille: Du patronage au réglage." Imaginaire social et représentations collectives. Éds. F. Dumont et Y. Martin. Québec: PU Laval, 1982. 335-46.

Lévesque, B. Le modèle québécois et le développement régional et local. Vers le néolibéralisme et la fin du modèle québécois? Montréal: Cahiers du CRISES, 1982.

Lévesque, B., G. L. Bourque, et Y. Vaillancourt. "Trois positions dans le débat sur le modèle québécois." Nouvelles pratiques sociales 12.2 (1999): 1-10. 
Lévesque, B. et Y. Vaillancourt. Les services de proximité au Québec. De l'expérimentation à l'institutionnalisation. Montréal: Cahiers du CRISES, 1998.

McCall, C. "Les murs de la cité: Territoires d'exclusion et espaces de citoyenneté." Lien social et politiques 34 (1995): 61-92.

McRoberts, K. "La thèse tradition-modernité: L'historique québécois." Les frontières de l'identité. Modernité et post-modernisme au Québec. Éds. M. Elbaz, A. Fortin, et G. Laforest. Québec: PU Laval-L'Harmattan, 1996. 29-45.

Melucci, A. "Société en changement et nouveaux mouvements sociaux." Sociologie et sociétés 10.2 (1978): 37-54.

-. "Sur le travail théorique d'Alain Touraine." Revue française de sociologie 16.3 (1975): 359-79.

Observatoire du Québec. L'urgence d'agir. Une économie interpellée par la mondialisation. Québec: PU Québec, 2002.

Piotte, J.-M. Du combat au partenariat. Interventions critiques sur le syndicalisme québécois. Montréal: Nota Bene, 1998.

Renaud, L., S. Chevalier, et J. O'Loughlin. “L'institutionnalisation des programmes communautaires: Revue des modèles théoriques et proposition d"un modèle." Revue canadienne de santé publique. 88.2 (1997): 109-13.

Schumpeter, J. A. Capitalism, Socialism and Democracy. London: Taylor \& Francis, 1976.

Shragge, E. Activism and Social Change: Lessons for Community and Local Organizing Action. Toronto: Broadview Press, 2003.

Thériault, J. Y. "De la critique de l'État à la reviviscence de la société civile." L'État aux orties? Éd. S. Paquerot. Montréal: Écosociété, 1996. 141-50.

Tardif, C. "Les corporations de développement communautaire au Québec: Processus d'institutionnalisation et trajectoires socioterritoriales spécifiques." Ph.D. diss., Université du Québec à Montréal, 2007.

Touraine, A. "Théorie et pratique d'une sociologie de l'action." Sociologie et sociétés 10.2 (1978): 149-88.

—. Pour la sociologie. Paris: Seuil, 1974.

—. Production de la société. Paris. Seuil,1973.

Vaillancourt, J.-G. "Deux nouveaux mouvements sociaux québécois: Le mouvement pour la paix et le mouvement vert." Le Québec en jeu: Comprendre les grands defies. Éds. G. Daigle et G. Rocher. Montréal: PU Montréal, 1992. 790-807. 
